Mechanics or Header

\title{
On a class of three-phase checkerboards with unusual effective properties
}

\author{
Richard V. Craster ${ }^{a}$ Sébastien Guenneau ${ }^{\mathrm{b}}$ Julius Kaplunov $^{\mathrm{c}}$ Evgeniya Nolde $^{\mathrm{c}}$ \\ ${ }^{a}$ Department of Mathematics, Imperial College London, London SW7-2AZ, UK \\ ${ }^{\mathrm{b}}$ Institut Fresnel, UMR CNRS 6133, University of Aix-Marseille, France \\ ${ }^{\mathrm{c}}$ Department of Mathematical Sciences, Brunel University, Uxbridge, Middlesex, UB8 3PH, U.K.
}

\begin{abstract}
We examine the band spectrum, and associated Floquet-Bloch eigensolutions, arising in a class of three-phase periodic checkerboards. On a periodic cell $\left[-1,1\left[^{2}\right.\right.$, the refractive index is defined by $n^{2}=1+g_{1}\left(x_{1}\right)+g_{2}\left(x_{2}\right)$ with $g_{i}\left(x_{i}\right)=r^{2}$ for $0 \leq x_{i}<1$, and $g_{i}\left(x_{i}\right)=$ 0 for $-1 \leq x_{i} \leq 0$ where $r^{2}$ is constant. We find that for $r^{2}>-1$ the lowest frequency branch goes through origin with linear behaviour, which leads to effective properties encountered in most periodic structures. However, the case whereby $r^{2}=-1$ is very unusual, as the frequency $\lambda$ behaves like $\sqrt{k}$ near the origin, where $k$ is the wavenumber. Finally, when $r^{2}<-1$, the lowest branch does not pass through the origin and a zero-frequency band gap opens up. In the last two cases, effective medium theory breaks down even in the quasi-static limit, while the high-frequency homogenization [Craster et al., Proc. Roy. Soc. Lond. A 466, 2341-2362, 2010] neatly captures the detailed features of band diagrams.

Keywords: Homogenization; Negative refraction; Acoustic band

\section{Résumé}

Sur une classe d'échiquiers à trois phases aux propriétés effectives singulières Nous étudions le spectre de bande associé aux modes de Floquet-Bloch dans une classe d'échiquiers périodiques. Sur une cellule de base $[-1,1[2$, l'indice de réfraction est défini par $n^{2}=1+g_{1}\left(x_{1}\right)+g_{2}\left(x_{2}\right)$ où $g_{i}\left(x_{i}\right)=r^{2} \quad$ (uneconstante), pour $\quad 0 \leq x_{i}<1$, et $g_{i}\left(x_{i}\right)=$ 0 pour $-1 \leq x_{i} \leq 0$. Pour $r^{2}>-1$, la première bande passe par l'origine avec un comportement linéaire, ce qui conduit à des propriété effectives rencontrées dans la plupart des structures périodique. En revanche, le cas $r^{2}=-1$ est moins ordinaire, puisque la bande de fréquences acoustiques $\lambda$ se comporte comme $\sqrt{k}$ au voisinage de l'origine, avec $k$ le nombre d'onde. Finallement, quand $r^{2}<-1$, la bande acoustique disparaît: la première bande ne passe plus par l'origine et une bande interdite à fréquence nulle apparaît. Dans ces deux derniers cas de figure, la théorie des milieux effectifs ne s'applique pas, alors que la théorie d'homogénéisation hautes fréquences [Craster et al., Proc. Roy. Soc. Lond. A 466, 2341-2362, 2010] reproduit avec précision les diagrammes de bandes.

Mots-clés : Homogénisation ; Réfraction négative ; Bande acoustique
\end{abstract}


Nous considérons un échiquier périodique dont la géométrie est décrite dans la figure 1. Les ondes optiques ou acoustiques qui s'y propagent sont solutions de l'équation de Helmholtz (1) dans les cases homogènes occupées par des matériaux isotropes qui sont positifs, nuls, ou négatifs. Par ailleurs, des conditions classiques de continuité de la composante tangentielle du champ électromagnétique (ou du champ de déplacement et de la composante normale du stress) sont imposées aux interfaces entre les différents matériaux de la cellule élémentaire et des conditions de Floquet-Bloch sur les bords opposés de celleci. Dans le cadre de la théorie d'homogénéisation hautes fréquences développée dans [1], nous nous intéressons plus particulièrement au cas où les conditions de Floquet-Bloch se réduisent à des conditions périodiques ou anti-périodiques, ce qui permet de déduire l'équation homogénéisée (4) qui conduit à une estimation fine des courbes de dispersion à travers la fréquence effective (5), pour des nombres d'onde au voisinage des points A, B et $\mathrm{C}$ de la zone de Brillouin, cf. figure 1. Il s'avère que l'on peut même déduire les relations de dispersion analytiquement dans le cas d'échiquiers à trois phases, données par (7) et (6).

Grâce à ces approches de type homogénéisation hautes fréquences [1], et Krönig-Penney [5], nous mettons en exergue trois types de comportements effectifs pour des ondes se propageant dans de tels échiquiers aux basses fréquences: ordinaire de type cristal photonique $\left(r^{2}>-1\right.$ : bande acoustique linéaire à l'origine, voir figure 3$\left.)(\mathrm{a})\right)$, singulier de type cristal plasmonique $\left(r^{2}=-1\right.$ : bande acoustique non-linéaire à l'origine, voir figure 2(a)) et de type métamatériaux $\left(r^{2}<-1\right.$ : perte de bande acoustique i.e. bande interdite à fréquence nulle, voir figure 2(b)).

Nous concluons notre étude par une application de type lentille échiquier par réfraction négative quand $r^{2}>-1$, avec l'image d'un point source à la fréquence donnée par l'estimation (8), voir figure 3(b).

\section{Introduction: Beyond effective medium theory with high-frequency homogenization}

Negative refraction is an emerging field in photonics introduced by Victor Veselago in the late 1960's [2], with renewed interest following the controversial claim of John Pendry that negative refraction makes a flat lens with unlimited resolution possible [3]. The quest for this superlens has fuelled research in structured photonic materials during the last decade. These composites are known as photonic crystals when the wavelength of light is of the same order as the typical heterogeneity size (Bragg frequency regime), and metamaterials when the wavelength is much larger (low frequency homogenization regime). The propagation of waves with anti-parallel group and phase velocities, an essential ingredient for negative refraction, was discussed in the late 1940's by Léon Brillouin [4].

In order to investigate the properties of photonic crystals, we consider the Helmholtz equation (1) in a periodic medium. This equation could, with appropriate notational and linguistic changes, hold for acoustic, electromagnetic, water or out-of-plane elastic waves and so encompasses many possible physical applications. We solve 


$$
\frac{\partial^{2} u}{\partial x_{1}^{2}}+\frac{\partial^{2} u}{\partial x_{2}^{2}}+\lambda^{2}\left[1+g_{1}\left(x_{1}\right)+g_{2}\left(x_{2}\right)\right] u=0
$$

for $u\left(x_{1}, x_{2}\right)$ on the square $-1<x_{1}, x_{2} \leq 1$, where $\lambda^{2}$ is the frequency squared. In the case of a three-phase checkerboard with a square cell as shown in Fig. 1, $g_{i}\left(x_{i}\right)$ is taken to be the piecewise constant

$$
g_{i}\left(x_{i}\right)=r^{2} \quad \text { for } \quad 0 \leq x_{i}<1 \text {, and } g_{i}\left(x_{i}\right)=0 \quad \text { for } \quad-1 \leq x_{i}<0 .
$$

We note that in the context of optics, the unknown $u$ in (1) stands for the longitudinal component of the electric field $E_{z}$ and $\lambda^{2}$ is associated with $\omega^{2} / c^{2}$ whereby $\omega$ is the electromagnetic wave frequency and $c$ is the speed of light in vacuum. Moreover, $\varepsilon_{r} \mu_{0}=$ $1+g_{1}\left(x_{1}\right)+g_{2}\left(x_{2}\right)$ where $\mu_{0}=1$ and $\varepsilon_{r}$ are the relative permeability and permittivity of the dielectric (non-magnetic) medium.

For waves through an infinite, perfect, doubly periodic checkerboard one can invoke Bloch's theorem [4] and simply consider the square cell with quasi-periodic Bloch boundary conditions applied to the edges:

$$
\begin{aligned}
& u\left(1, x_{2}\right)=e^{1 \kappa_{1}} u\left(-1, x_{2}\right), u_{x_{1}}\left(1, x_{2}\right)=e^{1 \kappa_{1}} u_{x_{1}}\left(-1, x_{2}\right), \\
& u\left(x_{1}, 1\right)=e^{1 \kappa_{2}} u\left(x_{1},-1\right), u_{x_{2}}\left(x_{1}, 1\right)=e^{1 \kappa_{2}} u_{x_{2}}\left(x_{1},-1\right),
\end{aligned}
$$

that involves the Bloch wave-vector $\boldsymbol{\kappa}=\left(\kappa_{1}, \kappa_{2}\right)$ characterizing the phase-shift as one moves from one cell to the next. There is also continuity of $u, u_{x_{1}}, u_{x_{2}}$ along $x_{1}=0$ and $x_{2}=0$. This Bloch problem is solved explicitly and dispersion relations that link the frequency and Bloch wavenumber are deduced; as is well-known in solid state physics [4] only a limited range of wavenumbers need be considered, namely the wavenumbers along the triangle shown in the reciprocal Brillouin lattice of Fig. 1 and these form the irreducible Brillouin zone. The dispersion curves reported in Fig. 2-3 illustrate several interesting features: stop-bands for which wave propagation is not possible, and regions of flat dispersion curves for which the group velocity is zero and features of slow sound or light occur. In this paper, we focus our attention to the lowest curves, associated with averaged properties of the checkerboards, and discuss the extension of existing effective theories to higher frequencies.

Conventional homogenization is widely assumed to be ineffective for modelling photonic crystals as it is limited to low frequencies when the wavelength is long relative to the microstructural lengthscales. Here the recently developed high frequency homogenization theory [1], which is free of the conventional limitations, is used to generate effective partial differential equations on a macroscale, that have the microscale embedded within them through averaged quantities. We focus our attention on periodic checkerboards, some of them with sign-shifting coefficients $[13,15]$ : the latter are known to support a host of surface plasmons associated with highly resonant features less than conducive for any effective theory. Our aim is actually to push the newly introduced high-frequency homogenization beyond its limits.

At leading order, the field $u(\mathbf{x})$ propagating inside the periodic checkerboard can be represented as $U(\boldsymbol{\xi}) f(\mathbf{X})$ where $\boldsymbol{\xi}$ and $\mathbf{X}$ are the short- and long-scales [1]. The function 

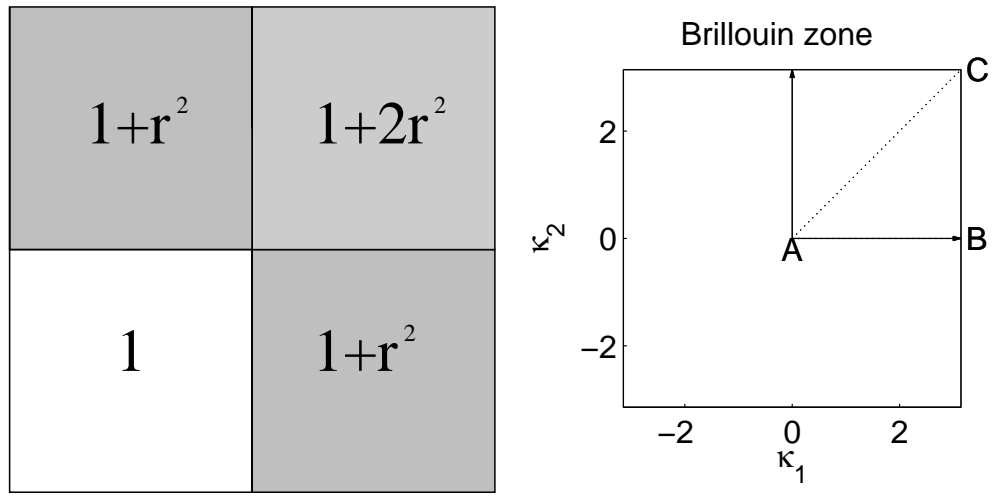

Figure 1. Left: A single cell for the checkerboard geometry, for piecewise constant media. Right: The reciprocal Brillouin lattice in wavenumber, $\kappa=\left(\kappa_{1}, \kappa_{2}\right)$, space

$U$ represents the local short-scale solution at a specific standing wave frequency, $\lambda_{0}$ (which is in the high frequency regime), and $f$ the long-scale variation. Ultimately an homogenized PDE emerges for $f$ entirely on the macroscale [1]

$$
T_{i j} \frac{\partial^{2} f}{\partial X_{i} \partial X_{j}}+\left(\lambda^{2}-\lambda_{0}^{2}\right) f=0
$$

where the spatially constant tensor $T_{i j}$ incorporates the short-scale information associated with the standing wave frequency $\lambda_{0} ; \lambda$ is the full frequency. The PDE can be augmented by additional terms if there is material variation leading to localised defect modes [7]. The PDE (4) can then be utilized for, say, Bloch waves where $f \sim \exp (1 \mathbf{k} \cdot \mathbf{X} / 2)$, to find local dispersion relations

$$
\lambda \sim \lambda_{0}\left(1+\epsilon^{2} \frac{T_{i j} k_{j}^{2}}{8 \lambda_{0}^{2}}\right)
$$

valid near the standing wave frequency $\lambda_{0}$; this formula is explicitly for those periodicperiodic standing waves at wavenumber $A$ and almost identical formulae hold for the other standing waves. Thus, the constant $T_{i j}$, once identified, completely encapsulate the effect of the microstructure on the dispersion properties of the system; the disparity of scales is encapsulated in $\epsilon$ which is assumed positive and $\ll 1$. Some typical values of the tensor $T_{i j}$ are given for the cases $r^{2}=-1$ and -2 in table 2 . One should note that in some cases the diagonal entries, are identical (effective isotropic parameters), different (anisotropic parameters) and even of opposite signs (a hallmark of anomalous dispersion).

\section{An exact dispersion relation for a class of three-phase checkerboards}

We have first investigated the dispersion curves associated with three-phase periodic checkerboards using the finite element software COMSOL MULTIPHYSICS. However, the convergence of the numerical algorithm can be hazardous, due to sign-shifting coefficients [15]. Thankfully, the Bloch problem for such checkerboards can be solved analytically and this provides probably the only non-trivial two dimensional structure with 


\begin{tabular}{ccccc}
\multicolumn{4}{c}{$r^{2}=-1$} \\
\hline & $\lambda$ & $\mu$ & $T_{11}$ & $T_{22}$ \\
$A$ & 0 & 0 & $N / A$ & $N / A$ \\
$A$ & 4.801 & 2.605 & -2.907 & 0.195 \\
$A$ & 4.801 & $2.605 \mathrm{i}$ & 0.195 & -2.907 \\
$C$ & 2.652 & 0 & -1.813 & -1.813 \\
$C$ & 4.519 & 2.345 & 2.951 & -0.380 \\
$C$ & 4.519 & $2.345 \mathrm{i}$ & -0.380 & 2.951 \\
$B$ & 2.336 & $0.767 \mathrm{i}$ & -3.445 & 1.394 \\
$B$ & 4.477 & $2.350 \mathrm{i}$ & 3.215 & 0.384 \\
$B$ & 4.821 & 2.595 & -0.199 & -2.774
\end{tabular}

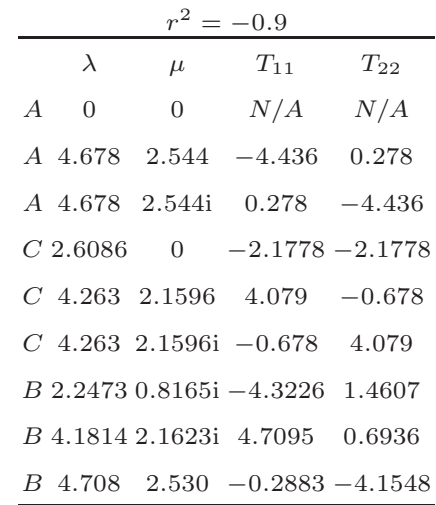

Table 1

The standing wave frequencies, $\lambda$, the $\mu$ and the corresponding $T_{11}$ and $T_{22}$ for the lowest three dispersion curves for $r^{2}=-1$ and $r^{2}=-0.9$

Table 2

\begin{tabular}{cccc}
\hline$\lambda$ & $\mu$ & $T_{11}$ & $T_{22}$ \\
$A 2.8898$ & 0 & 0.4519 & 0.4519 \\
$C 3.0217$ & 0 & -0.3412 & -0.3412 \\
$B 2.9632$ & 0.3524 & -0.41 & 0.3596 \\
\hline
\end{tabular}

Values of $\lambda$ and the corresponding $\mu$ at the wavenumbers for standing waves with the associated values of $T_{11}$ and $T_{22}$ for the lowest dispersion curve for $r^{2}=-2$.

an exact solution; it is a natural generalization of the classical Kronig-Penney [5] onedimensional piecewise constant periodic medium. The dispersion relation follows from setting $u\left(x_{1}, x_{2}\right)=Q_{1}\left(x_{1}\right) Q_{2}\left(x_{2}\right)$ in the governing equation (1). From the piecewise constant fonction (2), and from the Bloch conditions (3), the following coupled equations emerge [6]

$$
2 \gamma_{i} \beta_{i}\left(\cos \kappa_{i}-\cos \gamma_{i} \cos \beta_{i}\right)+\left(\beta_{i}^{2}+\gamma_{i}^{2}\right) \sin \gamma_{i} \sin \beta_{i}=0,
$$

for $i=1,2$. These are dispersion relations for $\lambda^{2}, \mu^{2}$ (the latter is a separation constant) in terms of $\kappa_{1}, \kappa_{2}$; the parameters $\gamma_{i}, \beta_{i}$ act to couple the equations as

$$
\beta_{i}^{2}=\lambda^{2} / 2 \mp \mu^{2}, \quad \gamma_{i}^{2}=\lambda^{2}\left(1 / 2+r^{2}\right) \mp \mu^{2},
$$

for $i=1,2$ with the minus, plus signs for $i=1,2$ respectively. This two variable dispersion relation is solved via matrix Newton iteration. For the exact solution found in this section, one finds $\lambda, \mu$ explicitly and some sample eigenvalues are given in table 2 . Notably some frequencies, $\lambda$, have two associated $\mu$ values, one real and one imaginary and these correspond to degenerate cases where the dispersion curves touch.

\section{Illustrative electromagnetic paradigms whereby effective medium theory fails}

Effective properties of periodic checkerboards are of particular mathematical interest, and their study goes back a long way, with the seminal paper by Keller [8] for twophase checkerboards, and those of Mortola-Steffe [9], Craster-Obnosov [10] and Milton [11] for four-phase checkerboards. Physicists have further analysed the effective properties of two-phase checkerboards with continuously varying parameters in the context of 
(a) $r^{2}=-1$

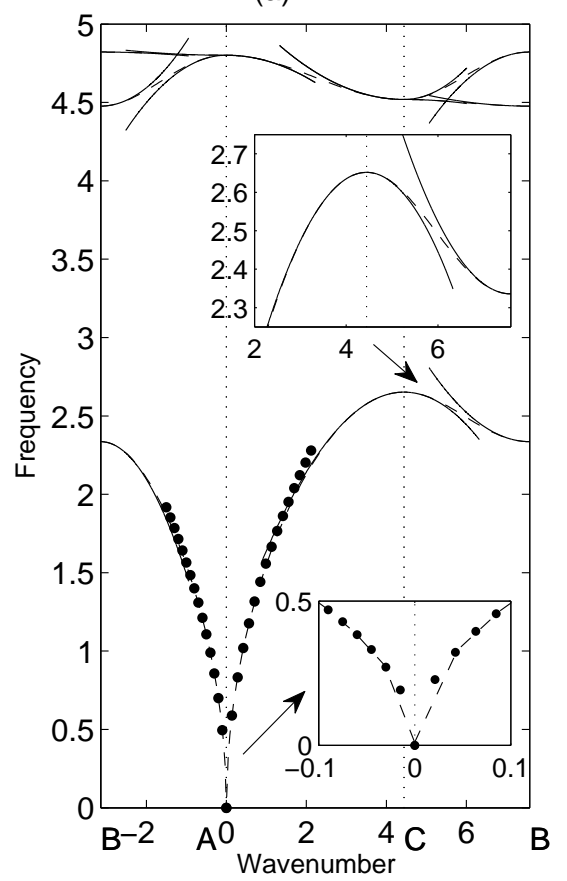

(b) $r^{2}=-2$

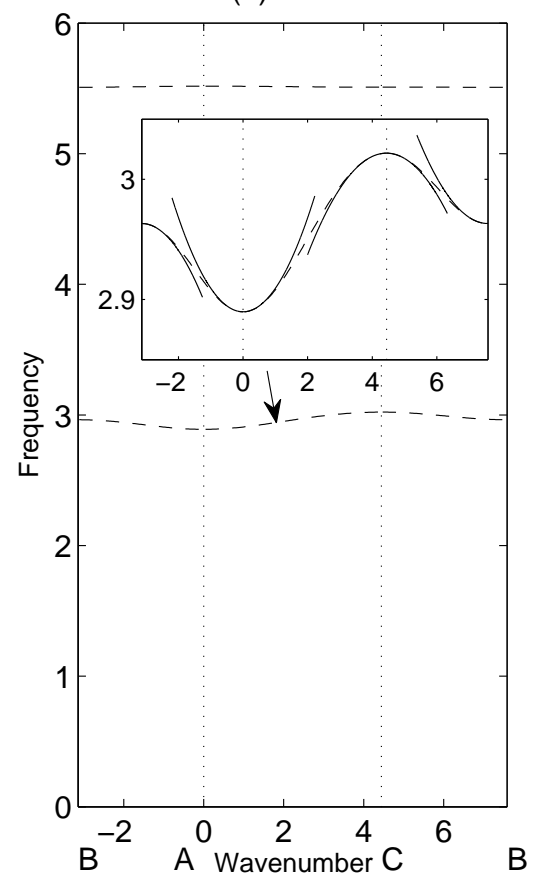

Figure 2. Dispersion diagrams for $r^{2}=-1$ (a) and $r^{2}=-2$ (b). Panel (a) shows the lowest three exact dispersion curves from the explicit dispersion relations and the asymptotic dispersion curves with the behaviour at the lowest curve that touches the origin shown dotted. The lower inset shows the detail near the origin comparing the exact (dashed) and asymptotic (dotted) solutions. The upper inset shows a similar comparison (asymptotics given by the solid line) for the lowest curve near wavenumber position $\mathrm{C}$. Panel (b) shows that there is no acoustic band and that the dispersion curves are nearly flat. The inset shows an enhanced view of the lowest dispersion branch with the numerics (dashed) shown together with asymptotics (solid).

photonic crystals [12]. In this note, we investigate the case of three-phase checkerboards with negative and vanishing refractive index (when $r^{2}$ takes negative values). Such a study is motivated by the fabrication of nano-scale gold and silver checkerboards whose cells alternate positive and negative refractive index media in the visible range of frequencies. Potential applications lie in extra-ordinary transmission of light through the sub-wavelength aperture holes in such checkerboards, and tremendously enhanced local density of states for light confinement [13]. These physical phenomena are underpinned by the effective properties of such checkerboards. Hence, an adequate homogenization model for such structures is of pressing importance.

We start to explore the case of a checkerboard with $r^{2}=-1$. Dispersion diagrams reported in figure 2(a) compare the solution from the exact dispersion relations with those from the asymptotics. It clearly demonstrates the superiority of the high-frequency homogenization approach which reproduces very precisely the acoustic and optical branches, thereby extending classical homogenization to the stop band (Bragg) regime of frequencies. Moreover, the region where the lowest branch cuts the origin, the long wave low frequency regime, is no longer linear and asymptotics of the dispersion relation show that the local behaviour is that $\lambda \sim 6^{1 / 4}|\kappa|^{1 / 2}$. The insets show detail of the asymptotics versus the exact solution.

Figure 2(b) shows the case of a checkerboard with $r^{2}=-2$; the acoustic band is lost 
for $r^{2}<-1$, which is reminiscent of singular problems in homogenization of periodic arrays of infinitely conducting fibres in transverse electric polarization (modelled with Dirichlet boundary conditions) [14]. In the present case, we do not consider Dirichlet conditions (corresponding to hard walls in acoustics) but we rather have sign-shifting conditions across some interfaces. It is clear that effective medium theory breaks down, however the high-frequency homogenization approach captures the fine features of the nearly flat dispersion curves, see the inset to figure 2(b), which are associated with slow waves, another topical subject in photonics.

We finally study the case of a checkerboard with $r^{2}=-0.9$, figure 3(a) looks visually similar to figure 2(a); however, a closer view on a log-log scale (not shown) of the acoustic band reveals that the long wave low frequency limit differs from that when $r^{2}=-1$. For $r^{2}=-0.9$ one gets the usual effective medium result with frequency linearly related to wavenumber however for $r^{2}=-1$ the effective medium theory no longer holds and a different asymptotic relation holds $\left(\lambda \sim 6^{1 / 4}|\kappa|^{1 / 2}\right)$; we further observe that the highfrequency homogenization approach (dotted line) captures the fine features of this curve (dashed line).

Figure 3(a) also shows the light-line emerging from $C$ relevant for waves impinging upon a checkerboard structure rotated through $\pi / 4$. Notably the light-line crosses the lowest dispersion curve in such a way that their group velocities are opposite and therefore one can induce negative refraction at this frequency. The asymptotic theory explicitly identifies this frequency as

$$
\lambda=\lambda_{0} / \sqrt{1-T / 4}
$$

where $T_{11}=T_{22}=T$ for that case. The estimate is that $\lambda \sim 2.1$ and computations for this are shown in figure 3 .
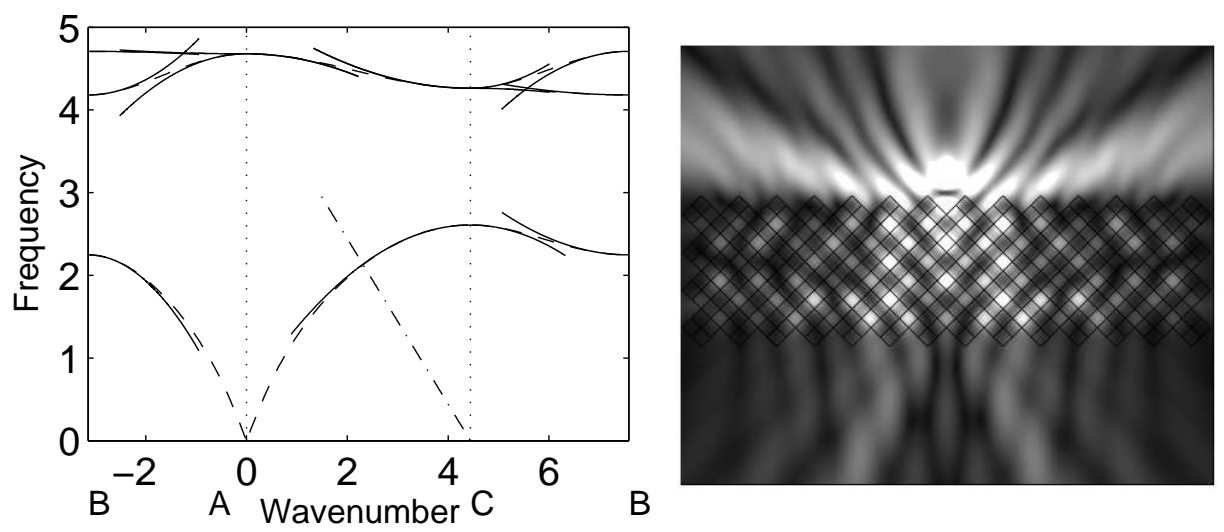

Figure 3. Dispersion diagrams for $r^{2}=-0.9$. Left panel shows the dispersion curves that are visually close to those for $r^{2}=-1$ however, the behaviour near $A$ for low frequency is asymptotically different between these cases. The dot-dash line is the light-line for waves impinging upon the rotated checkerboard. The right panel shows the response due to a harmonic point source at frequency $\lambda=2.1$ above a finite size checkerboard for $r^{2}=-0.9$ with each cell rotated by an angle $\pi / 4$; it displays an image below the checkerboard in accordance with the Snell-Descartes law for a negative refractive index. 


\section{Conclusions}

We have investigated the Bloch spectrum of periodic, three-phase, checkerboards. The focus has been on special cases in which the refractive index squared (i.e. the relative permittivity in the contex of optics) in homogeneous cells can take vanishing and/or negative values, and we have found that anomalous dispersion effects of waves are possible, including acoustic bands with non linear features for vanishing wavenumbers, zero frequency stop bands, and nearly flat dispersion curves. The last two items are known to be respectively associated with singularly perturbed problems in electromagnetism that cannot be homogenized in a conventional way [14], as no wave is allowed to propagate in the periodic structure at low frequencies, and slow waves, whereby delay lines can be achieved.

\section{References}

[1] R.V. Craster, J. Kaplunov, and A. V. Pichugin, Proc. Roy. Soc. Lond. A 466, 2341-2362, 2010

[2] V.G. Veselago, Sov. Phys. Uspekhi 10, 509-514, (1968).

[3] J.B. Pendry, Phys. Rev. Lett. 86, 3966, (2000).

[4] L. Brillouin, Wave propagation in periodic structures, McGraw-Hill, (1946).

[5] R. L. Kronig and W. G. Penney. Quantum mechnics in crystals lattices Proc. Roy. Soc. Lond. 130, pp. 499-531 (1931)

[6] R.V. Craster, J. Kaplunov, E. Nolde and S. Guenneau, submitted 2010

[7] E. Nolde, R. V. Craster, and J. Kaplunov (2010), under review.

[8] J. B. Keller, J. Math. Phys. 5, 548 (1964).

[9] S. Mortola and S. Steffe, Atti Accad. Naz. Linei Rend Cl. Sci. Fis. Mat. Natur. 78,77 (1985).

[10] R. V. Craster and Y. V. Obnosov, SIAM J. Appl. Math. 61, 1839 (2001).

[11] G. W. Milton, J. Math. Phys. (2001).

[12] F. Zolla and S. Guenneau, Phys. Rev. E 67, 026610 (2003).

[13] S. Guenneau and S. A. Ramakrishna, C. R. Physique 10, 352 (2009).

[14] C.G. Poulton, L.C. Botten, R.C. McPhedran, N.A.P. Nicorovici and A.B.Movchan, SIAM J. Appl. Math. 61, 1706-1730 (2001).

[15] A-S Bonnet-BenDhia, P.Ciarlet Jr. and C.M. Zwolf J. Comput. Appl. Math 234(6), 1912-1919 (2010). 